\title{
SOME REMARKS ON PENMAN'S EQUATIONS FOR THE EVAPOTRANSPIRATION
}

\author{
J. A. BUSINGER \\ Institute of Horticultural Engineering, Wageningen. Ministry of Agri- \\ culture, Holland
}

\section{SUMMARY}

Special attention is paid to the empirical factors in Penman's equations for the evapotranspiration. It is proposed to use the more theoretical expression of PENMAN for $E_{T}$ practically. For this purpose a theoretical formulation of the factor $f(u)$ is given; the quantity $\boldsymbol{E}_{o}$ which leads easily to misunderstanding, is omitted and the empirical factor $a$ is replaced by another empirical factor $\varepsilon$. It was found that $\varepsilon=0.92$ gave the best fit to the observations.

Penman (1948) derived the well-known equations for the evapotranspiration :

$$
\begin{aligned}
& E_{T}=a E_{o} \\
& \text { and } E_{o}=\frac{\Delta H_{0}+\gamma}{\Delta+\gamma} \frac{E_{a}}{\Delta+\gamma}=\frac{\Delta H_{0}+f(u)\left(e_{a}-e_{d}\right)}{\Delta+\gamma}
\end{aligned}
$$

where $e_{a}=$ saturation vapour pressure;

$\boldsymbol{e}_{\mathfrak{d}}=$ vapour pressure ;

$E_{T}=$ potential evapotranspiration

$E_{0}=$ evaporation of a water surface ;

$E_{a}=f(u)\left(e_{a}-e_{d}\right)$;

$f(u)=$ factor, dependent on wind velocity, surface roughness and stability of the air layer;

$H_{0}=$ net gain of radiation at a water surface ;

$u=$ wind velocity;

$a=$ empirical factor ;

$\gamma=$ psychrometer constant

$\triangle=$ slope of saturation vapour pressure curve.

In these equations the quantity $E_{0}$ is related to the evaporation of a water tank, to make it possible to estimate the factor $\alpha$.

The theoretical derivation of $E_{0}$ is not based on the evaporation of a tank but on the evaporation of a water surface with the same roughness as its surroundings and without heat capacity. The theoretical $E_{o}$ is fitted to the measured $E_{o}$ by an empirical relation for $f(u)$. This empirical relation holds only for the conditions of Penmav's experiments and must be estimated again for every case with other conditions (see MakKINK, 1955).

Therefore it is better to eliminate $E_{o}$ in the calculation of $E_{T}$ and to use a formula of the kind Penman and Schofield (1952) have given:

$$
E_{T}=\begin{gathered}
\Delta H_{T}+\gamma E_{a} \\
\Delta+\gamma / S D
\end{gathered}
$$

where $H_{T}=$ net gain of radiation at the surface,

$S=$ stomatal factor,

and $D=$ daylength factor. 
More general and for practical purposes we may write:

$$
E_{T}=\frac{\Delta H_{T}+\gamma E_{a}}{\Delta+\varepsilon \gamma}
$$

where $\varepsilon$ is an empirical factor, which replaces $a$ in Eq. (1).

To estimate $\varepsilon$ it is necessary to have direct measurements of $E_{T}$ and a theoretical founded relation $f(u)$.

Building on the similarity of the exchange of heat, water vapour and momentum we find:

$$
f=\frac{C_{P} \varrho}{\gamma P} \frac{u}{\left(u / u_{*}\right)^{2}}
$$

where $C_{P}=$ heat capacity of the air,

$$
\begin{aligned}
& P=59 \mathrm{cal} / \mathrm{mm} \mathrm{H}_{2} \mathrm{O} \mathrm{cm}{ }^{2}, \\
& u_{*}=\text { friction velocity. }
\end{aligned}
$$

$u / u_{*}$ is determined by the velocity profile and is depending on the roughness and the stability of the air layer. For neutral conditions holds :

so we find for Eq. (5)

$$
\frac{u}{u_{*}}=\frac{1}{k} \ln \frac{z+z_{0}-d}{z_{0}}
$$

$$
f=\frac{k^{2} C_{P} \varrho}{\gamma P}-\frac{u}{\ln \frac{z+z_{o}}{z_{0}}-d}
$$

where $k=\mathrm{v}$. KARMAN's constant $=0.4$,

$d=$ zere point displacement by the vegetation, and $z_{0}=$ roughness parameter.

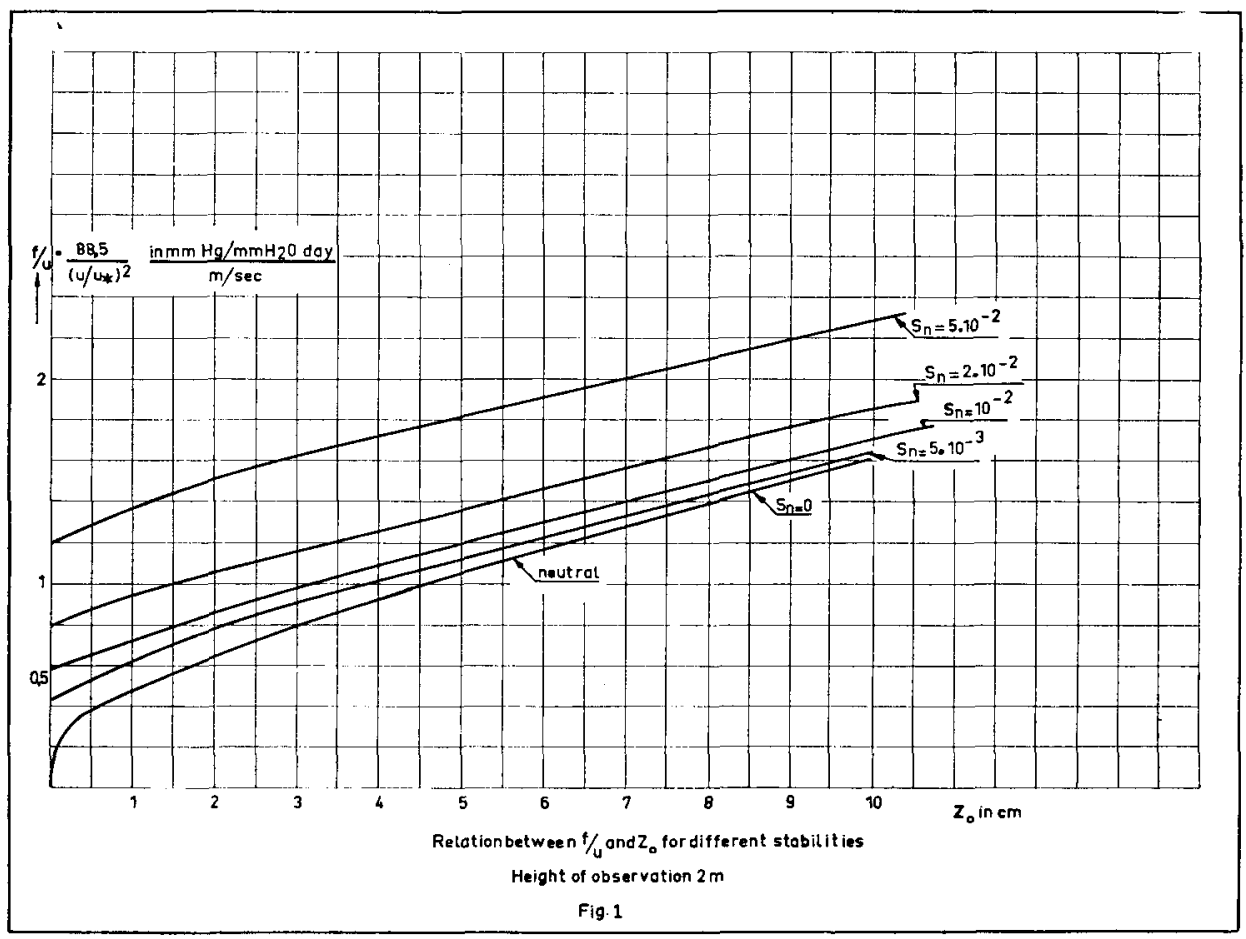


In Fig. 1 the relation between $f / u$ and the surface roughness is given. In this figure also curves are drawn for different stability numbers $S n$, a quantity introduced by Businger (1954).

$$
S n=\frac{g K z_{0}}{u^{3}{ }_{*} \bar{T} C_{P} \varrho}
$$

where $g=$ acceleration due to gravity,

$$
\begin{aligned}
& K=\text { flux of sensible heat, } \\
& \bar{T}=\text { mean temperature in }{ }^{\circ} K .
\end{aligned}
$$

It is rather difficult to estimate $S n$ from observations because a complete heat balance of the surface is required. In general there can be said that $S n$ is negligible at windvelocities above $5 \mathrm{~m} / \mathrm{sec}$.

The influence of $S n$ becomes very important on clear days with no or light winds.

When $z_{0}=1 \mathrm{~cm} ; u_{2}=1 \mathrm{~m} / \mathrm{sec}$ and $K=0.4 \mathrm{cal} / \mathrm{cm}^{2} \mathrm{~min}$; then $\mathrm{Sn}=$ $3.10^{-2}$. At this value of $S n$ the factor $f$ is more than twice as large as under neutral conditions. So it is desirable to consider the correction for stability as well as possible. The curves for $\mathrm{S} n$ in Fig. 1 are calculated with the theoretical assumptions Businger (1954) made.

An estimation of $\varepsilon$ is made with the aid of a number of observations from the lysimeter station at Wageningen, which Mr. MakKink put at my disposal.

\begin{tabular}{|c|c|c|c|c|c|c|c|c|}
\hline Date & $f / u$ & $\begin{array}{r}\Delta H_{T} \\
+\gamma E_{a}\end{array}$ & $\triangle+\varepsilon \gamma$ & $E_{T}$ calc. & $E$ meas. & $\triangle E_{T}$ & Prec. & $\bar{t}$ \\
\hline 1954 & & & $\varepsilon=0.92$ & $\mathrm{~mm} / \mathrm{day}$ & $\mathrm{mm} / \mathrm{day}$ & $\mathrm{mm} / \mathrm{day}$ & $\mathrm{mm}$ & ${ }^{\circ} \mathrm{C}$ \\
\hline $10 / 5$ & 0.40 & 10.3 & 1.58 & 6.5 & 5.7 & 0.8 & & 21 \\
\hline $11 / 5$ & 0.50 & 8.4 & 1.52 & 5.5 & 5.0 & 0.5 & & 20 \\
\hline $13 / 5$ & 0.42 & 10.2 & 1.35 & 7.6 & 8.1 & -0.5 & & 17 \\
\hline $14 / 5$ & 0.45 & 6.3 & 1.17 & 5.4 & 6.4 & -1.0 & & 13 \\
\hline $21 / 5$ & 0.42 & 4.1 & 1.03 & 4.0 & 2.8 & 1.2 & 1.7 & 9 \\
\hline $25 / 5$ & 0.60 & 9.8 & 1.46 & 6.7 & 7.0 & -0.3 & & 19 \\
\hline $28 / 5$ & 0.50 & 13.3 & 1.58 & 8.4 & 7.4 & 1.0 & & 21 \\
\hline $1 / 6$ & 0.60 & 1.6 & 1.21 & 1.3 & 0.8 & 0.5 & 7.1 & 14 \\
\hline $2 / 6$ & 0.60 & 2.5 & 1.21 & 2.1 & 2.8 & -0.7 & 0.4 & 14 \\
\hline $3 / 6$ & 0.60 & 5.1 & 1.35 & 3.8 & 3.7 & 0.1 & 6.5 & 17 \\
\hline $4 / 6$ & 0.65 & 9.6 & 1.41 & 6.8 & 7.8 & -1.0 & & 18 \\
\hline $11 / 6$ & 0.30 & 2.8 & 1.17 & 2.4 & 1.9 & 0.5 & 1.3 & 13 \\
\hline $14 / 6$ & 0.30 & 2.6 & 1.06 & 2.5 & 2.8 & -0.3 & 0.1 & 10 \\
\hline $15 / 6$ & 0.40 & 5.7 & 1.26 & 4.5 & 4.7 & -0.2 & & 15 \\
\hline $16 / 6$ & 0.35 & 4.2 & 1.35 & 3.1 & 2.5 & 0.6 & 0.3 & 17 \\
\hline $21 / 6$ & 0.35 & 2.3 & 1.26 & 1.8 & 2.5 & -0.7 & 1.5 & 15 \\
\hline $23 / 6$ & 0.40 & 3.75 & 1.26 & 3.0 & 3.5 & -0.5 & & 15 \\
\hline $24 / 6$ & 0.45 & 7.2 & 1.35 & 5.3 & 6.4 & -1.1 & & 17 \\
\hline $29 / 6$ & 0.45 & 2.8 & 1.13 & 2.5 & 2.7 & -0.2 & & 12 \\
\hline $6 / 7$ & 0.50 & 2.9 & 1.10 & 2.7 & 2.3 & 0.4 & 2.4 & 11 \\
\hline $7 / 7$ & 0.55 & 3.3 & 1.11 & 3.0 & 2.4 & 0.6 & 1.1 & 11.5 \\
\hline $8 / 7$ & 0.90 & 5.8 & 1.26 & 4.6 & 4.5 & 0.1 & 0.1 & 15 \\
\hline $9 / 7$ & 1.0 & 6.2 & 1.30 & 4.8 & 4.8 & 0.0 & & 16 \\
\hline $13 / 7$ & 0.25 & 1.1 & 1.19 & 0.9 & 0.6 & 0.3 & 0.8 & 13.5 \\
\hline $14 / 7$ & 0.30 & 1.7 & 1.23 & 1.4 & 0.3 & 1.1 & 7.7 & 14.5 \\
\hline
\end{tabular}

The best fit was obtained, using $\varepsilon=0.92$, see Table 1 .

Table 1. 
It may be expected that the empirical constant $\varepsilon$ is more general valid and has more scientific value than the constant $\alpha$.

\title{
REFERENCES
}

Businger, J. A.: Some aspects of the influence of the earth's surface on the atmosphere. Med. Verh. K.N.M.I., Serie B nr. 60, 1954.

Makkink, G. F.: Toetsing van de berekening van de evapotranspiratie volgens Penman. Landbouwk. Tijdschrift 67 (1955) 267-282.

Peniran, H. L. : Natural evaporation from open water, bare soil and grass. Proc. Roy. Soc. A, 193 (1948) 120-146.

- - and R. K. SchоғдеLD: Some physical aspects of assimilation and transpiration. S.E.B.Symposium nr. 5 (1951) 115-12.9.

\section{EVAPOTRANSPIRACION DE LOS CULTIVOS EN ESPANA SU DETERMINACION POR MEDIO DE LISIMETROS}

\author{
LUIS CAVANILLAS \\ Instituto Español de Edafologia y Fisiologia Vegetal y \\ Instituto Nacional de Investigaciones Agronómicas, Madrid, España
}

\section{CONCLUSIONES}

De todo lo que tan concisamente hemos expuesto sobre nuestros trabajos y experiencias con lisímetros de sistema gravimétrico deducimos las conclusiones siguientes :

1a) Los lisímetros de sistema gravimétrico son los más recomendables cuando se trate de conocer con exactitud y al detalle las intensidades de evapotranspiración de las plantas.

2a) Este sistema de lisímetros requiere un estudio muy cuidadoso y delicado del régimen de aportes de agua y fertilizantes, para conseguir la mayor semejanza posible entre la vegetación de las plantas en campo abierto y en lisímetros. Si este estudio no es suficientemente acertado, los resultados pueden ser muy erróneos.

3a) Es interesante tambien adoptar algun tipo de lisímetro monolítico y de mayores dimensiones, para las plantas de mayor porte y para perfeccionamiento del sistema gravimétrico, en general para toda clase de cultivos.

Desde que se dió en España el primer impulso de importancia al desarrollo de nuevos regadíos, creándose las Confederaciones Sindicales Hidrográficas, se sintió la necesidad de conocer lo más exactamente posible, las exigencias en agua de los distintos cultivos en las diferentes regiones del país.

Con esta finalidad, la Confederación Hidrográfica del Ebro, estableció un equipo de 100 lisimetros, anejo a la Estación de Estudios de Aplicación de Riegos, en Binefar (Huesca). Terminada la instalación en el año 1935, solo pudo funcionar entonces pocos meses, hasta el año 1936 en que, al producirse la guerra civil española, quedaron paralizados los trabajos en aquella Estación, reanudándose desde el año 1940.

Fueron alentadores los primeros resultados que iban obteniéndose y tanto el Consejo Superior de Investigaciones Científicas, a traves de su Instituto de Edafología y Fisiología Vegetal, como el Instituto Nacional de Investigaciones Agronómicas, estimaron de interés la creación de una red de Estaciones de lisímetros. Y así en colaboración ambos Institutos, fueron estableciendo sucesi- 